\title{
Effects of Dynamic Bedroom Lighting On Measures of Sleep and Circadian Rest-Activity Rhythm in Inpatients With Major Depressive Disorder
}

Canazei Markus ( $\nabla$ markus.canazei@uibk.ac.at)

University of Innsbruck

Weninger Johannes

Bartenbach $\mathrm{GmbH}$

Pohl Wilfried

Bartenbach $\mathrm{GmbH}$

Marksteiner Josef

Regional Psychiatric Hospital

Weiss Elisabeth

University of Innsbruck

\section{Research Article}

Keywords: dawn-dusk simulation, blue-depleted light, actigraphy, sleep, circadian activity rhythm, major depression

Posted Date: October 13th, 2021

DOI: https://doi.org/10.21203/rs.3.rs-955553/v1

License: (c) (i) This work is licensed under a Creative Commons Attribution 4.0 International License. Read Full License 


\section{Abstract}

Bright light therapy is an effective treatment option for seasonal and non-seasonal affective disorders. However up to now, no study has investigated effects of dynamic bedroom lighting in hospitalized patients with major depression.

A bedroom lighting system, which automatically delivered artificial dawn and dusk and blue-depleted nighttime lighting (DD-N lighting) was installed in a psychiatric ward. Patients with moderate to severe depression were randomly assigned to stay in bedrooms with the new lighting or standard lighting system. Patients wore wrist actimeters during the first two treatment weeks. Additionally, hospitalization duration and daily psychotropic medication were retrieved from patients' medical charts.

Data from thirty patients were analyzed. Patients under DD-N lighting generally woke up earlier $(+20$ minutes), slept longer (week 1: +11 min; week 2: +27 min) and showed higher sleep efficiency $(+2.4 \%)$ and shorter periods of nighttime awakenings (-15 minutes). In the second treatment week, patients started sleep and the most active 10-hour period earlier (-33 min and -64 min, respectively).

This pilot study gives first evidence that depressed patients' sleep and circadian rest/activity system may benefit from adjunctive bedroom lighting when starting inpatient treatment.

\section{Introduction}

Altered alertness and disturbed circadian rhythms as well as sleep problems are often reported in person with affective disorders ${ }^{1}$ and may play a crucial role in the pathogenesis of this disease ${ }^{2}$.

Light acutely alters vigilance in humans ${ }^{3}$. Timed light exposure additionally has the potential to resynchronize the circadian clock ${ }^{4}$ and to improve sleep quality ${ }^{3}$. Thus, timed light exposure is a promising non-pharmacological intervention option in the treatment of affective disorders.

Today, light therapy is a first-line treatment for seasonal affective disorders ${ }^{5}$ (SAD), and may be used in treating patients with moderate to severe depression ${ }^{6,7}$. Moreover, combined medication and light therapy showed greater efficacy than pharmacotherapy alone in depressed patients ${ }^{6}$. Despite these promising results, light therapy is still underused in clinical settings today.

Light therapy comprises a daily morning, polychromatic white light exposure of 5000 lux-hours, is fastacting in its antidepressant effect ${ }^{1,8}$, and has a minor side-effect profile (e.g., headache, eyestrain, nausea, and agitation) occurring in up to $45 \%$ of patients only within the first treatment days ${ }^{9}$.

In 1989, Terman and colleagues ${ }^{10}$ conducted a pioneering study and reported beneficial effects of artificial dawn and dusk in three SAD patients. Later, two landmark studies provided evidence that artificial dawn is as effective as light therapy in the treatment of $S A D^{11,12}$. 
Dawn simulators are installed at the bed head-side and deliver timed, gradually increasing light intensities in the early morning. They differ in several technical aspects to light therapy devices: light exposure occurs during the sleep-wake transition period over a time span of 30 minutes to 3 hours and light intensities are gradually rising and peaking at the time of planned awakening with 250 to 1,000 lux (applied light dose in dawn simulation is thus substantially lower than in light therapy). Dawn simulators are further perceived as a more natural light intervention, deliver light without side-effects, and thus may be easier and more convenient to use than light therapy devices ${ }^{13}$.

Research in healthy people has broadened our understanding of artificial dawn effects: it improves sleep inertia $^{14-16}$ and cognitive performance after awaking ${ }^{15,17}$. In addition, artificial dawn induces some physiological effects, e.g., a phase advance of the melatonin rhythm ${ }^{18}$, an accelerated decline in skin temperature ${ }^{16}$ and a reduced heart rate gradient during sleep-wake transition ${ }^{19}$.

Besides dawn, dusk is a second important natural cue for photic entrainment feeding into the evening oscillator of the biological clock ${ }^{20}$. Although the pioneering study of Terman and colleagues ${ }^{10}$ used artificial dawn and dusk, effects of artificial dusk were scarcely investigated. To the best of our knowledge only Danilenko and Hommes ${ }^{21}$ have compared effects of a rectangular lights on/off pulse with a dusk simulation in the evening in healthy subjects, showing reduced body movements in the first 40 minutes of sleep but no phase shifting of the circadian clock under artificial dusk.

Moreover, up to now, only two field studies have investigated effects of a combined artificial dawn and dusk intervention 22,23 . In these studies, persons with dementia living in nursing homes were exposed to artificial dawn and dusk that was fitted to individual sleep times. In a first study ${ }^{22}$, persons with dementia had an earlier sleep onset, shorter sleep latency, longer sleep duration and decreased nighttime activity after three intervention weeks. In a second study ${ }^{23}$, patients with dementia reported better mood upon awakening under artificial dawn and dusk. However, in this study no effects were observed on actigraphically measured circadian activity rhythm and sleep parameters.

In addition to light therapy and artificial dawn and dusk, nocturnal lighting may affect humans. Research has shown that blue-depleted nighttime lighting may reduce nighttime arousal ${ }^{24}$ and minimize disruptions of circadian and sleep parameters in healthy people $25-27$.

It is worth noting, that blue-depleted nighttime lighting can also be generated by wearing blue-light blocking glasses. In a recently published systematic review ${ }^{28}$ it was shown that this intervention decreased manic symptoms in bipolar patients but delivered inconclusive results in improving mood in patients with major depression. However, clear evidence was found in this review of blue-light blocking glasses for a reduction in sleep latency in patients with sleep disorders.

So far, research has shown beneficial effects of artificial dawn in SAD patients and of artificial dawn and dusk in patients with dementia. No study has yet investigated effects of a dawn-dusk simulation and blue-depleted nighttime lighting in hospitalized patients, in particular in patients with affective disorders. 
The present prospective pilot study closes this gap. Outcome measures were actigraphically recorded sleep and circadian rest-activity rhythm parameters and clinical health data (i.e., medication and length of hospitalization). Based on current knowledge, we hypothesized an improved sleep quality (i.e., shorter sleep onset latency and higher sleep efficiency) and more stable circadian rest-activity cycles in hospitalized patients residing under this dynamic bedroom lighting.

\section{Methods}

\subsection{Bedroom lighting}

\subsubsection{Bedrooms with artificial dawn-dusk and blue-depleted nighttime lighting (DD-N lighting)}

In two double bedrooms in a psychiatric ward, seven tunable-white LED luminaires (WS14-01 FFF LED wallwasher, Projektleuchten $\mathrm{GmbH}$, Dortmund, Germany) were installed at the upper wall opposite to the patients' beds (see Supplementary Materials S1). Each luminaire comprised 24 LEDs (12 Luxeon Z-ES 2200 Kelvin and 12 Luxeon Z-ES 4000 Kelvin). In addition, bed-side reading lights (research prototypes, Projektleuchten $\mathrm{GmbH}$, Dortmund Germany), containing 10 LEDs (Luxeon Z-ES 2200 Kelvin), were installed in the bedrooms as well (see Supplementary Materials S2). To guarantee a consistent lighting design for the whole private area of the patient, the adjacent bathroom lights were further equipped with tunable-white LED luminaires (research prototypes, Projektleuchten $\mathrm{GmbH}$, Dortmund Germany), providing the same light colors at the same daytime period as the bedroom lights. Ambient bedroom and bathroom luminaires were DALI-dimmable and controlled by a central lighting control system (Tridonic $\mathrm{GmbH}$ Co KG, Dornbirn, Austria). The reading light was manually adjustable in its intensity by a dimmer installed nearby the bed.

The lighting control strategy was aligned to the daily life of the patients in the psychiatric ward. Specifically, patients leave their beds at 07:00h (or were woken by the staff at the latest at 07:00h), so daytime activities (e.g., morning toilet and dressing) typically start at 07:00h, followed by breakfast at 7:30h (served in common rooms outside the bedrooms). Typically, patients reenter their bedrooms during short therapy breaks in the morning and afternoon and after lunch, but stay in public clinic areas during the day otherwise. The bedroom is again the main place of residence until after dinner at around 18:00h. Commonly, patients get into their beds between 20:00h and 21:00h and read, watch TV or relax. Usually, patients start sleeping after the last caring procedures is finished at 22:00h.

In line with these social routines and to generate a smooth light-induced sleep-wake transition period by ambient bedroom lighting, in the present study artificial dawn began 35 minutes before daily activities started (at 06:25h) and was implemented with the ambient lighting system. During artificial dawn, light intensities gradually increased from 0 to 209 lux (horizontally at bed's height) accompanied by risen color temperatures from 2192 Kelvin to 3588 Kelvin. At 07:00h, the light intensity and light color was at its 
maximum. To stop artificial dawn, patients had to leave their beds, as the light switch was installed at the entrance of the bedroom.

During the day (from 07:00h to 20:00h), ambient bedroom lighting must be switched manually and provided 209 lux at bed's height with a color temperature of 3588 Kelvin. After 20:00h, ambient room lights were again dimmed automatically (artificial dusk) in its illuminance and color temperature over a period of 2 hours. At 22:00h, room lights provided 100 lux at bed's height with a color temperature of 2192 Kelvin. In case, patients preferred to use reading lights for zonal illumination of the bed-side area, they were able to stop the artificial dusk at any time. Reading lights provided 20 lux at bed's height with 2192 Kelvin and could be manually dimmed.

If necessary, room lights could be switched on during the night at any time and provided 100 lux at bedside level with 2192 Kelvin.

The lighting control strategy is graphically summarized in Figure 1. A comprehensive summary of photometrical measures generated by the DD-N lighting system at specific periods in time, is given in Table 1.

\subsubsection{Standard bedroom lighting}

Lighting design in the standard double bedrooms followed European lighting standards for hospitals (EN DIN 12464-1) and was implemented with two customary luminaires (,Pureline Basic', Zumtobel AG, Dornbirn, Austria), which were installed at the wall above the patient's head. Each luminaire housed two fluorescent lamps (Osram T16 39W/840 G5 HO). Reading lights comprised the same fluorescent lamp technology and offered 52 lux horizontally (measured at the height of the bed). Following recommendation from Spitschan ${ }^{29}$, photometrical measures are again summarized in Table 1.

\subsubsection{Dimensions of the bedrooms and daylight penetration in the bedrooms}

All four study bedrooms (two bedrooms with DD-N lighting and standard lighting, respectively) had the same room area (length: 4.60m; width: $2.9 \mathrm{~m}$ ), were located on the second floor and oriented to the east. Each bedroom had one window with an area of approximately 1.60 square meters. Tall, densely grown trees reduced daylight levels in the bedrooms and direct sunlight penetration in the morning. The daylight factor (i.e., the amount of daylight at 1-meter distance from the window in the bedroom on overcast days) in all four bedrooms was low $(1.2 \% \pm 0.8)$. 
Table 1

Photometric characterization of the lighting interventions.

\section{Standard bedroom DD-N lighting lighting}

Lighting system

Timing

$\begin{array}{lll}\begin{array}{l}\text { Ambient } \\ \text { light }\end{array} & \begin{array}{l}\text { Reading Ambient light } \\ \text { light }\end{array}\end{array}$

$\begin{array}{ll}07: 00 h & 21: 00 h \\ - & - \\ 21: 00 h & \substack{07: 00 h \\ \star}\end{array}$

Color properties

correlated color

temperature

color rendition (CRI)

CIE coordinates $(x, y)$

$3513 \quad 3513 \quad 2192$

Kelvin

86

$(0.41$,
$0.40)$
Kelvin

86

$(0.41$

$0.40)$
$06: 25 \mathrm{~h}-$

07:00h 07:00h 20:00h
Reading light 20:00h -

22:00h 22:00h -

$06: 25 h$

Illuminances (mean $\pm \mathrm{SD}$;

Ix)

\begin{tabular}{lllllll} 
horizontal, at bed level & $140 \pm$ & $52 \pm 4$ & $20 \pm 2$ & $209 \pm 21$ & $100 \pm 10$ & $20 \pm 2$ \\
& 14 & & & & & \\
$\begin{array}{l}\text { vertical, at eye level of } \\
\text { patient - 20 tilted }\end{array}$ & $131 \pm$ & $48 \pm 5$ & $19 \pm 2$ & $196 \pm 23$ & $94 \pm 11$ & $19 \pm 2$ \\
$\begin{array}{l}\text { vertical, at eye level of } \\
\text { patient }-70^{\circ} \text { tilted }\end{array}$ & $131 \pm$ & $57 \pm 7$ & $20 \pm 2$ & $208 \pm 21$ & $98 \pm 11$ & $20 \pm 2$ \\
\hline
\end{tabular}

Luminances (range;

$\mathrm{cd} / \mathrm{m}^{2}$ )

$\begin{array}{lllllll}\text { at opposite wall } & 29-41 & 5-23 & 3-10 & 27-98 & 15-49 & 3-10 \\ \text { at ceiling } & 21-345 & 5-98 & 2-75 & 25-780 & 13-394 & 2-75\end{array}$

a-opic equivalent daylight (D65) illuminance (Ix)

\begin{tabular}{|lllllll|}
\hline S-cone-opic & 63.20 & 21.76 & 2.83 & 88.29 & 14.13 & 2.83 \\
\hline M-cone-opic & 129.69 & 44.66 & 14.32 & 178.58 & 71.59 & 14.32 \\
\hline L-cone-opic & 151.71 & 52.25 & 20.70 & 208.52 & 103.49 & 20.70 \\
\hline rhodopic & 92.04 & 31.70 & 7.73 & 129.94 & 38.66 & 7.73 \\
\hline melanopic & 77.10 & 26.55 & 5.88 & 112.83 & 29.41 & 5.88 \\
\hline
\end{tabular}

Note: spectral irradiances and illuminances were measured with a spectroradiometer (specbos 1211-2, JETI Technische Instrumente GmbH, Jena, Germany) and color properties were calculated with the 
software JETI LiVAl; luminances were measured with a calibrated CANON EOS 650D camera with fisheye optic; the alpha-opic irradiances were derived from the spectral irradiance measurements excel-toolbox S026-2018 provided by the CIE; * typical switching times of ambient lights and reading lights were determined from staff's observations; tabulated light spectra are given in Supplementary Materials Table S1 and Figure S3.

\subsection{Outcome Measures 2.2.1 Actigraphy}

The rest-activity cycle is a core behavioral measure of the circadian system and often disturbed in depressed patients ${ }^{30}$. An actimeter is a body-worn device, which comprises an accelerometer and allows the measurement of physical activity over periods of days or weeks. By means of these activity data sleep quality parameters ${ }^{31}$ and circadian activity rhythm parameters ${ }^{32}$ can be calculated. Due to the fact, that sleep parameters derived from actimetry are similar to polysomnography (validation shows high sensitivity but moderate to low specificity ${ }^{33}$ ), this method has often been employed in sleep and light impact research ${ }^{34}$.

In the present study, we used a wrist-worn, light-weight (19 grams), water-resistant device (ActiGraph wGT3x-BT; Pensacola, FL, USA). The device was worn for the first two treatment weeks and recorded three-dimensional activity data with a sample rate of $30 \mathrm{~Hz}$. Additionally, the device included a light sensor, which measured illuminances at wrist level of up to 5,000 lux. To minimize non-wearing times, the actimeter was attached with a non-removable textile bracelet. We used the software ActiLife (Version 6.8.1) to retrieve and process actimetry data (i.e., we aggregated data over time periods of 1 minute) and to run the Cole-Kripke sleep scoring algorithm. For the sleep analysis, usually a daily bed time protocol is needed. In the present study, bed times were estimated from activity data: the individual starting time in bed was defined as the beginning of the latest 10-min period between 20:00h to 23:00h with lower physical activity compared to the mean daytime activity level of the subject; the individual end time in bed was defined as the end of the earliest 10-min period between $06: 00 \mathrm{~h}$ to 8:00h with lower activity compared to the mean daytime activity level of the ongoing day. The following sleep parameters were derived for each night: total sleep time (TST; hours), sleep onset latency (SOL; minutes), sleep efficiency (SE; percent), number of wake episodes (WASO; count data) and sleep onset and offset times (clock times). For statistical analysis, we calculated mean values of these sleep parameters separately for the first and second treatment week. To derive circadian activity rhythm parameters, we applied a nonparametric analysis routine ${ }^{35}$ to weekly activity data and calculated the following parameters also separately for each of the two treatment weeks: intra-daily variability (IV), inter-daily stability (IS), relative amplitude (RA), most active 10-hour period (M10), clock time when M10 starts (M10 onset), least active 5-hour period (L5), and clock time when L5 starts (L5 onset).

To compare light exposure levels between the two intervention groups, daytime-specific light exposure levels were additionally retrieved from the wrist-worn actimeters. 


\subsubsection{Hospital stay and psychotropic medication}

Length of hospitalization, daily non-pharmacological treatments (i.e., ergotherapy, physiotherapy, and psychotherapy) and psychiatric medications (i.e., antidepressant, antipsychotic, and sedative medication) were retrieved from the patients' medical chart. To compare treatments with different antidepressants, we calculated equivalent doses referring to Citalopram ${ }^{36}$. A similar procedure was applied to antipsychotic medication ${ }^{37}$ and sedative medication ${ }^{38}$ with the reference agents Chlorpromazine and Diazepam, respectively. Due to the fact, that pro re nata (PRN) medication was used occasionally (on 28 out of 420 treatment days) in few study participants (DD-N lighting: $n=4$ in the first week and $n=4$ in the second week; standard lighting: $n=2$ in the first week and $n=4$ in the second week), we did not extract PRN medication data from patients' health records.

\subsection{Study protocol and data collection procedure}

Clinical effects of light therapy can be seen in SAD patients after the first treatment week ${ }^{13,39}$. Even for patients with non-seasonal depression, light therapy induces a strong reduction of symptoms within two weeks ${ }^{6}$. Based on these results and the fact, that depressed patients stayed at the psychiatric ward for an average of 17.5 days \pm 7.9 , the participants continuously wore a wrist actimeter on their non-dominant hands from the first day of admission up to fourteen days.

Study participants were patients of a psychiatric hospital with an ICD-10 diagnosis of affective disorder (F32, F33, and F34; ICD-1040). Moreover, patients had to suffer from moderate to severe depression, quantified with a Beck Depression Inventory score (BDI-V) ${ }^{42}$ of at least 35 at admission. Moreover, inpatient treatment should last at least for 14 days. Patients showing acute suicidal ideation at admission were excluded from study participation.

Written informed consent was given by all subjects prior to study inclusion. The study protocol strictly followed the guidelines outlined in the 1964 Declaration of Helsinki and was approved by the ethical committee of the Medical University of Innsbruck, Austria.

In total, 187 patients were asked for study participation at admission by a psychiatrist over the course of one year. Many of these patients did not meet inclusion criteria or declined to participate $(n=129)$. Consequently, 58 patients were randomly assigned to the two interventions (DD-N lighting: $n=29$; standard lighting: $n=29$ ). Data from 15 patients were lost to follow-up (due to unwillingness to wear the actimeter; usually after a wearing period of one or two days). Finally, thirteen patients had shorter inpatient treatment periods than 14 days and thus were excluded from analysis. Finally, data from 30 patients were subjected to analyses. A detailed study flow diagram of the present study is given in Figure 2.

\subsection{Statistical Analysis}

Descriptive statistics are given as mean and standard deviation (mean \pm SD) and figures show averages and $95 \%$ confidence intervals of means. To compare demographic variables, length of hospitalization, 
and non-pharmacological treatments between the two intervention groups either a t-test, chi-squared test or exact Fisher test were run. Two-way mixed analysis of variances (ANOVAs) with the between-subjects factor light intervention and the within-subjects factor treatment week were used for the analysis of actimetric parameters, antidepressant and antipsychotic medication, and light exposure. Due to the fact, that data of equivalent doses of sedative medication differed on the first treatment day, a mixed ANOVA, controlled for the equivalent dose of sedative medication on the first treatment day, was run. Effect sizes (partial eta-squared) are given for significant effects. All analyses were run with a significance level of 0.05 (two-sided).

By means of the software package $\mathrm{G}^{*}$ Power $^{43}$ (Version 3.1.9.4), a sensitivity analysis for a repeatedmeasures, within-between interaction model was run. For this model and under usual statistical assumptions (power $=80 \%$, alpha $=0.05$, correlation among repeated measurements $=0.5$, and nonsphericity correction epsilon $=1$ ), a sample size between 16 to 34 subjects allows the detection of moderate intervention effects ${ }^{44}$. This effect size was also found in light therapy studies with people with depressive disorders ${ }^{6,7}$.

\section{Results}

A total of 16 and 14 patients stayed in bedrooms with standard lighting and DD-N lighting, respectively. Gender distribution, mean age, distribution of ICD-10 diagnoses, and mean depression score (BDI-V) did not differ between the two groups (see Table 2). 
Table 2

Description of study sample.
Patients in bedrooms with standard lighting
Patients in bedrooms with DD-
N lighting ( $n=14)$
Statistics
$(n=16)$

Gender

Female

5

8

$p=.153$

Male

11

6

Age (years)

$44.6 \pm 10.4$

$48.4 \pm 14.1$

$p=.363$

ICD-10 diagnoses

F32 (depressive

2

1

$p=.125$

episode)

F33 (recurrent

depressive episode)

F34 (persistent mood 7

disorder)

BDI-V

$62.2 \pm 17.0$

$57.2 \pm 14.0$

$p=.444$

Note: values are either shown as mean \pm standard deviation or count data; BDI-V = Beck Depression Inventory; ICD-10 = International Classification of Diseases ${ }^{40}$.

\subsection{Sleep-related effects}

\subsubsection{Total sleep time (TST)}

We found a large interaction effect between the two factors light intervention and treatment week on TST, $F(1,28)=6.650, p=.015$, partial $\eta^{2}=.192$. In the first week, TST was significantly higher under DD-N lighting (493.96 min \pm 13.11 ) compared to standard lighting (483.37 min \pm 13.08 ), with a mean difference of $11 \mathrm{~min}, \mathrm{~F}(1,28)=4.880, \mathrm{p}=.036$, partial $\eta^{2}=.148$. In the second week, TST was again significantly higher under DD-N lighting ( $504.15 \mathrm{~min} \pm 26.28)$ compared to standard lighting (477.58 min \pm 18.62 ), with a mean difference of $27 \mathrm{~min}, \mathrm{~F}(1,28)=10.408, \mathrm{p}=.003$, partial $\eta^{2}=.271$. In addition, TST significantly increased over time under DD-N lighting only, $F(1,13)=5.476, p=.036$, partial $\eta^{2}=.296$.

\subsubsection{Sleep onset latency (SOL)}

We could not observe any intervention effect on SOL, all $p>.10$.

\subsubsection{Sleep efficiency (SE)}


A mixed ANOVA could reveal a significant main effect of intervention on $\mathrm{SE}, \mathrm{F}(1,28)=7.626, p=.010$, partial $\eta^{2}=.214$, with higher SE under DD-N lighting $(92.59 \% \pm 2.82)$ compared to standard lighting $(90.18 \% \pm 2.91)$. The interaction effect and main effect of measurement time was not significant, both $p$ $>.10$.

\subsubsection{Wake after sleep onset (WASO)}

WASO was significantly lower under DD-N lighting (38.70 min \pm 12.71 ) compared to standard lighting (54.14 $\mathrm{min} \pm 11.97$ ) across both treatment weeks, with a mean difference of $15 \mathrm{~min}, \mathrm{~F}(1,28)=23.557, p<$ .001 , partial $\eta^{2}=.968$. Statistical analysis did not yield a significant interaction nor main effect of time on WASO, both $p>.10$.

\subsubsection{Sleep onset time}

There was a statistically significant interaction between the factors light intervention and time of measurement on sleep onset time, $F(1,28)=4.312, p=.047$, partial $\eta^{2}=.133$. In the first week, sleep onset times did not differ between DD-N lighting $(21: 48 \mathrm{~h} \pm 41 \mathrm{~min})$ and standard lighting $(21: 59 \mathrm{~h} \pm$ $23 \mathrm{~min}), \mathrm{p}>.10$. However, in the second week sleep onset time was significantly earlier under DD-N lighting $(21: 28 \mathrm{~h} \pm 28 \mathrm{~min})$ than under standard lighting $(22: 01 \mathrm{~h} \pm 33 \mathrm{~min})$, with a mean difference of 33 $\min , \mathrm{F}(1,28)=8.642, p=.007$, partial $\eta^{2}=.236$. In addition, patients under DD-N lighting started sleep earlier in week 2 compared to week $1, F(1,13)=5.428, p=.037$, partial $\eta^{2}=.295$.

\subsubsection{Sleep offset time}

Independent of the treatment week, patients woke up earlier in the morning under DD-N lighting (06:35h \pm $20 \mathrm{~min})$ compared to standard lighting $(06: 55 \mathrm{~h} \pm 24 \mathrm{~min})$, with a mean difference of $20 \mathrm{~min}, \mathrm{~F}(1,28)=$ $8.141 ; p=.008$, partial $\eta^{2}=.225$. We further observed no significant interaction and main effect of measurement time on sleep offset time, both $p>.10$.

Interaction plots for all sleep parameters are given in the Supplementary Materials S4. In addition, sleep data for the first three treatment days were compared between the two intervention groups (see Supplementary Materials S5), indicating that actimetric sleep data was comparable between the two groups within the first inpatient treatment days.

\subsection{Circadian rest-activity rhythm effects \\ 3.2.1 Intra-daily Variability (IV) and Inter-daily Stability (IS)}

We could not observe any intervention effect on IV and IS, all $p>.10$.

\subsubsection{Relative Amplitude (RA)}

RA significantly differed between the two treatment weeks, $\mathrm{F}(1,28)=7.229, p=.012$, partial $\eta^{2}=.205$, with lower RA in the week $1(0.937 \pm 0.030)$ compared to week $2(0.946 \pm 0.026)$. The interaction effect and main effect of intervention on RA was not significant, both $p>.10$. 


\subsubsection{Most active 10-hours (M10)}

Similar to RA, we could reveal a significant time of measurement effect on M10, $F(1,28)=27.127, p<$ .001 , partial $\eta^{2}=.492$, with lower M10 in week 1 (2108 \pm 572$)$ compared to week 2 (2455 \pm 788$)$. Again, the interaction effect and main effect of intervention on M10 was not significant, both $p>.10$.

\subsubsection{Onset time of M10}

A two-factor mixed ANOVA showed a significant interaction between the factors light intervention and measurement time on the onset time of $\mathrm{M} 10, \mathrm{~F}(1,28)=9.305, p=.005$, partial $\eta^{2}=.249$. Onset times of M10 did not differ in treatment week $1, p>.10$, but in treatment week $2, \mathrm{~F}(1,28)=11.793, p=.002$, partial $\eta^{2}=.296$, with earlier onset time under DD-N lighting $(06: 43 \mathrm{~h} \pm 32 \mathrm{~min})$ compared to standard lighting (07:47h $\pm 63 \mathrm{~min})$, a mean difference of $64 \mathrm{~min}$. In addition, under both bedroom lighting systems, onset time of M10 changed significantly over time: for standard lighting the onset time was delayed in week 2 (07:47h $\pm 63 \mathrm{~min})$ compared to week $1(07: 13 \mathrm{~h} \pm 57 \mathrm{~min}), \mathrm{F}(1,15)=4.608, p=.049$, partial $\eta^{2}=.235$. In contrast, under DD-N lighting M10 onset time significantly advanced from treatment week $1(07: 12 \mathrm{~h} \pm$ $61 \mathrm{~min})$ to treatment week $2(06: 43 \mathrm{~h} \pm 32 \mathrm{~min}), \mathrm{F}(1,13)=5.297, p=.039$, partial $\eta^{2}=.289$.

\subsubsection{Least active 5-hours (L5)}

We could not reveal any intervention effect on $L 5$, all $p>.10$.

\subsubsection{Onset time of L5}

Interaction between the factors light intervention and measurement time on onset time of L5 reached significance, $F(1,28)=4.250, p=.049 ;$ partial $\eta^{2}=.132$. Post-hoc analyses revealed no differences in the onset times of $L 5$ between the two interventions in treatment week $1, p>.10$, but a significant difference in treatment week $2, F(1,28)=4.321 ; p=.047$, partial $\eta^{2}=.134$, with an earlier onset time under DD-N lighting $(23: 10 \mathrm{~h} \pm 55 \mathrm{~min})$ compared to standard lighting $(23: 49 \mathrm{~h} \pm 46 \mathrm{~min})$, with a mean difference of 39 min. Under both interventions, onset times of L5 did not differ between the two treatment weeks: both $p>$ .10 .

In the Supplementary Materials S6a and S6b, interaction plots for effects on circadian rest-activity rhythm parameters are shown.

\subsection{Length of hospitalization}

The length of hospitalization was not different between the two interventions: standard lighting (21.4 days \pm 3.4 ) and DD-N lighting (20.3 days \pm 5.8$), \mathrm{t}(28)=1.074, p=.851$.

\subsection{Pharmacotherapy}

\subsubsection{Antidepressant medication}


An independent-samples t-test was run to determine if there were differences in antidepressant medication on the first treatment day between the two intervention groups. The equivalent daily dose of Citalopram did not differ at the beginning of inpatient treatment for patients under DD-N lighting (20.82 $\mathrm{mg} \pm 14.13)$ and standard lighting (20.74 $\mathrm{mg} \pm 14.12), p>.10$, see Figure 3.

Moreover, patients were prescribed a similar mean daily equivalent dose of Citalopram across the 14 treatment days with an average of $27.67 \mathrm{mg} \pm 13.68$ under DD-N lighting and $27.59 \mathrm{mg} \pm 14.18$ under DD-N lighting, $p>.10$. The daily equivalent dose of Citalopram, however, increased from $35.46 \mathrm{mg} \pm$ 29.67 in the first treatment week to $40.07 \mathrm{mg} \pm 35.40$ in the second treatment week, $\mathrm{F}(1,28)=4.453 ; p=$ .044 ; partial $\eta^{2}=.137$. We could not reveal a significant interaction between the factors light intervention and measurement time, $p>.10$.

\subsubsection{Antipsychotic medication}

Ten patients in bedrooms with DD-N lighting and 9 patients in bedrooms with standard lighting were treated with antipsychotic medication during the first two treatment weeks. Those patients were prescribed a similar mean daily equivalent dose of Chlorpromazine of $105.95 \mathrm{mg} \pm 64.36$ under DD-N lighting and $109.96 \mathrm{mg} \pm 89.59$ under standard lighting, $p>.10$. Again, neither the interaction between the two factors nor the main effect of measurement time of daily equivalent doses of Chlorpromazine were significant, both $p>.10$.

\subsubsection{Sedative medication}

Fourteen patients in each intervention group were treated with Benzodiazepines across the 14 treatment days. About half of this type of medication (43-51\%) was given daily in the evening and night (17:0022:00h). An independent-samples t-test showed that the equivalent daily dose of Diazepam were significantly different at the first treatment day in patients under DD-N lighting ( $7.44 \mathrm{mg} \pm 8.44)$ compared to standard lighting $(20.03 \mathrm{mg} \pm 17.89), t(26)=9.264, p=.025$. Thus, a mixed ANOVA, controlled for the equivalent dose of Diazepam at treatment day 1 , was run. We could not reveal a difference between the two light intervention groups in the daily equivalent dose of Diazepam (DD-Nlighting: $7.56 \mathrm{mg} \pm 6.21$; standard lighting: $8.71 \mathrm{mg} \pm 6.11 ; p>.10$ ). In addition, the interaction between the two factors light intervention and measurement time were not significant, $p>.10$. However, we observed a main effect of time with a significant higher daily dose of Diazepam in the first treatment week $(10.91 \mathrm{mg} \pm 4.16)$ compared to the second treatment week $(5.37 \mathrm{mg} \pm 4.77), \mathrm{F}(1,26)=28.650 ; p<$ $.001 ;$ partial $\eta^{2}=.534$.

\subsection{Confounder analysis}

\subsubsection{Non-pharmacological treatments}

Following a multimodal treatment approach, all subjects took part in ergo-therapies, physiotherapies, and psychotherapies on work days from 09:00h to $11: 00 \mathrm{~h}$ and 14:00h to 16:00h. The daily mean number of therapies did not differ between the two groups over the 14 treatment days; DD-N lighting: 2.5 therapies \pm 
0.5 and standard lighting: 2.5 therapies $\pm 0.4, \mathrm{t}(28)=0.185, p=.767$. Additionally, both groups participated equally in the therapies: DD-N lighting: $20 \%$ ergo-therapy, $69 \%$ physiotherapy, and $11 \%$ psychotherapy; standard lighting: 18\% ergo-therapy, $71 \%$ physiotherapy, and $11 \%$ psychotherapy, $p=$ .882 .

\subsubsection{Light exposure across different daytime periods 3.5.2.1 Light exposure during the artificial dawn period (06:25h to 7:00h)}

As expected, mean illuminance level at wrist level was higher under DD-N lighting during automatic dawn $(43.86 \mathrm{Ix} \pm 6.00)$ compared to standard lighting $(20.47 \mathrm{Ix} \pm 5.6), \mathrm{F}(1,28)=8.099, p=.008 ;$ partial $\eta^{2}=.224$. We could not find a significant interaction nor main effect of time of measurement on illuminance in the early morning, both $p>.10$.

\subsubsection{Daytime light exposure (07:01 h to $21: 59 \mathrm{~h})$}

Mean daytime wrist illuminance levels did not differ $(p>.10)$ and were $200.41 \mathrm{~lx} \pm 72.80$ for subjects under DD-N lighting and $212.37 \mathrm{~lx} \pm 70.07$ under standard lighting. In addition, daily exposure duration above 1000 lux were also equivalent between the two groups; DD-N lighting: 50 min \pm 14 ; standard lighting: $49 \min \pm 13, p>.10$.

\subsubsection{Light exposure during the artificial dusk period (20:00h to 22:00h)}

Wrist illuminance levels were significantly lower under DD-N lighting (24.32 Ix \pm 28.31$)$ compared to standard lighting $(48.93 \mathrm{~lx} \pm 33.60)$ during the dusk period, $\mathrm{F}(1,28)=6.826, p=.014$; partial $\eta^{2}=.196$. The interaction effect and main effect of treatment week did not reach significance, both $p>.10$.

\subsubsection{Nighttime light exposure (22:01h to 06:24h)}

Following our expectations, nighttime light levels were significantly lower under DD-N lighting $(0.61 \mathrm{~lx} \pm$ 1.73) compared to standard lighting $(1.63 \mathrm{Ix} \pm 1.95)$ in the first treatment week, $p=.003$, and also in the second treatment week; DD-N lighting: $0.81 \mathrm{~lx} \pm 2.34$; standard lighting: $2.16 \mathrm{~lx} \pm 2.41, p=.019$. The interaction and time effects on nighttime illuminance measured at wrist level did not reach significance, both $p>.10$.

\section{Discussion}

The present study aimed at investigating dynamic ambient bedroom lighting effects in moderately to severely depressed patients at the beginning of inpatient treatment using wrist actigraphy and data from patients' medical charts. As expected, the bedroom lighting system significantly increased morning (from 6:25h to 7:00h) and decreased evening and nighttime light exposure (from 20:00h to 6:25h) compared to standard bedroom lighting. In addition, we observed sleep-related and circadian activity rhythm effects 
generated by the dynamic ambient bedroom lighting system. Those effects were likely not caused by differences in pharmacological and non-pharmacological inpatient treatments in the two intervention groups.

Under dynamic lighting, the timing of sleep phases was altered. Patients generally woke up earlier by 20 minutes and, with a delay of one week, also started sleep 33 minutes earlier. Moreover, and independent of treatment time, depressed patients showed shorter waking periods during nighttime sleep by 15 minutes under dynamic lighting and slept 11 minutes longer in treatment week 1 and 27 minutes longer in treatment week 2.

Sleep disturbances are highly prevalent in patients with depressive disorders. Research has also shown a bidirectional association between sleep disturbances and depression ${ }^{45}$. Thus, improving sleep at the beginning of treatment is of high importance ${ }^{46}$.

In a recently published review which summarized effects of antidepressants in depressed patients, Tazawa and colleagues ${ }^{47}$ could show that a successful pharmacotherapy is accompanied with sleeprelated effects such as shortened sleep onset latency and increased sleep efficiency (i.e., reduced nighttime activity levels and waking periods). In the present study dynamic lighting generated acute sleep-related effects within the first two inpatient treatment weeks.

Research further showed that depressive disorders are associated with circadian disturbances such as a delayed circadian rhythm ${ }^{48}$. In addition, a delayed peak in daytime activity is typically found in phases of acute depression ${ }^{49}$. One suspected mechanism of action of morning light therapy is its potential to phase advance the circadian system ${ }^{2}$. This effect has already been shown in people with seasonal affective disorder ${ }^{50}$. However, to date there is inconclusive evidence that phase shifting of circadian rhythms is the mechanism of action in the treatment of non-seasonal depression ${ }^{1}$.

In the present study, with some delay (in the second treatment week), the onset times of the daily most active and less active periods were earlier under dynamic bedroom lighting indicating that the intervention not only has influenced nighttime sleep but also circadian activity rhythm parameters. It should be mentioned, however, that sleep problems and circadian disturbances are mutually dependent ${ }^{51}$ and thus reported light effects on the circadian activity rhythm can hardly be separated from light effects on nighttime sleep parameters.

Our study found no effects on inter-daily stability and intra-daily variability of activity rhythms, which is in line with results from a systematic review of Burton and colleagues ${ }^{52}$. It can be assumed, that hospitalization and consequently the social rhythm imposed to patients' daily activities may have masked potential light effects on the stability and variability of daily physical activity cycles.

Altered physical activity is a core feature of depression ${ }^{46}$ and depressed patients often show lower daytime activity levels ${ }^{49,52}$ and a damped circadian activity profile ${ }^{46}$. During the course of treatment, 
these physical activity parameters usually improve ${ }^{52}$. We found similar results in our study. Irrespective of bedroom lighting, patients' daytime physical activity level and their daily activity rhythm amplitude improved over time, indicating a general inpatient treatment response.

In this study, patients under dynamic and standard bedroom lighting were prescribed equivalent daily doses of antidepressant and antipsychotic medication. Moreover, by controlling for group differences at the first treatment day, both groups also did not differ in sedative medication across the fourteen treatment days.

It is well documented, that only about half of all depressed patients respond to antidepressant medication and about one third experience remission of symptoms ${ }^{53}$. Moreover, clinical response to antidepressant medication can often be observed after weeks ${ }^{54}$, which is problematic for adherence, particularly because significant side effects of antidepressant medication (e.g., digestive problems, appetite disturbances, sleep problems, dizziness, or agitation) frequently occur from the beginning of pharmacotherapy. Consequently, supplementary interventions with a fast antidepressant response are needed.

For a more immediate relief of specific symptoms in depressed patients (e.g., distress, sleeplessness and restlessness), sedatives and antipsychotics are additionally prescribed ${ }^{55,56}$. It was recently shown, that a combined therapy (benzodiazepines + antidepressants) improve depression severity in the early phase of treatment (1-4 weeks) compared to antidepressants alone ${ }^{57}$. In addition, benzodiazepines ameliorate symptoms of insomnia efficiently. However, administration must be balanced judiciously against possible harms ${ }^{58}$.

There are also well recognized non-pharmacological interventions with immediate response in antidepressant treatment, such as electroconvulsive therapy ${ }^{59}$ and partial or total sleep deprivation ${ }^{60}$. However, these treatments are complex and can also have significant side effects. Due to its fast-acting response profile, light therapy has been discussed as further, well-tolerated treatment option for depressed patients ${ }^{6}$. The most recently published meta-analysis confirms the effectiveness of light therapy in non-seasonal depression but also states that more research is needed in severely depressed patients ${ }^{61}$. The present study, for the first time, provides initial evidence of a fast response in physical activity parameters of depressed inpatients to ambient light treatment.

We did not observe an intervention effect on the length of hospitalization. To date, research has clearly shown that increased sunlight exposure during the day reduced inpatient treatment duration in severely depressed patients ${ }^{62-65}$. It can be hypothesized that in our study the light intervention took place at a different time of day and thus may have not affected the length of hospitalization. Further studies are warranted to substantiate this assumption.

This study has several limitations. First, sample size was small. Although we included 58 patients in our study, only data from 30 subjects could be analyzed. Main cause of the high drop-out rate was the fact, 
that the wrist-worn actimeter was not well accepted. Moreover, a few patients reported that the actimeter disrupted nighttime sleep. Second, no further objective and subjective sleep measure (e.g., polysomnography, questionnaires) nor circadian phase marker (e.g., melatonin or core body temperature) were recorded to confirm results obtained from wrist actimetry. Third, self-rated depression symptoms were recorded at admission only. Fourth, actimetric data were only available after admission. However, by analyzing actimetry data within the first three treatment days, we could not observe differences in sleep parameters between the two intervention groups.

To conclude, in this pilot study first beneficial effects of dynamic bedroom lighting (artificial dawn and dusk and blue-depleted nighttime lighting) on inpatients with moderate to severe depression were reported. The light intervention showed fast-acting effects on sleep and circadian activity rhythm parameters which cannot be explained by differences in medication, non-pharmacological treatments and daytime light exposure. Reported effects indicate that ambient bedroom light may have significant short-term effects in inpatient treatment of persons with major depression. Results further complement research on light therapy in non-seasonal depression. Larger studies are warranted to confirm results from this pilot study and to establish dynamic ambient lighting as an effective additional treatment option.

\section{Declarations}

\section{Acknowledgments}

We are obliged to the staff (Reingard Falch, doctor in charge, and Eva Singewald, clinical psychologist) for recruiting study participants and conducting clinical screenings at admission. Furthermore, we want to thank Kathrin Bauernhofer for organizing on-site data collection and all patients for participating voluntarily in this study.

\section{Competing Interests Statement}

The authors declare no conflicts of interest.

\section{Funding}

The study was financially supported by the Austrian Research Promotion Agency (Grant No. 850747).

\section{Authors' contributions}

CM: developed study design, analyzed data, and drafted manuscript; WJ: programmed lighting control strategy, processed actimetry data and medication data, and analyzed data; PW: responsible for lighting design and conducted photometric measurements; JM: developed study design and supervised staff at the psychiatric ward; WE: developed study design, acted as principal investigator; all authors carefully revised the manuscript. 


\section{Data Availability Statement}

Aggregated data is provided by the corresponding author at reasonable request.

\section{References}

1. Cunningham, J. E. A., Stamp, J. A. \& Shapiro, C. M. Sleep and major depressive disorder: a review of non-pharmacological chronotherapeutic treatments for unipolar depression. Sleep Med, 61, 6-18 (2019).

2. Lee, H. J. Is Advancing Circadian Rhythm the Mechanism of Antidepressants? Psychiatry Investig. 16(7), 479-483(2019).

3. Faulkner, S. M., Bee, P. E., Meyer, N., Dijk, D. J. \& Drake, R. J. Light therapies to improve sleep in intrinsic circadian rhythm sleep disorders and neuro-psychiatric illness: a systematic review and meta-analysis. Sleep Med Rev, 46, 108-123 (2019).

4. Menculini, G. et al. Depressive mood and circadian rhythms disturbances as outcomes of seasonal affective disorder treatment: A systematic review. J Affect Disord, 241, 608-626 (2018).

5. Terman, M. \& Terman, J. S. Light therapy for seasonal and nonseasonal depression: efficacy, protocol, safety, and side effects. CNS Spectr, 10 (8), 647-663 (2005).

6. Geoffroy, P. A., Schroder, C. M., Reynaud, E. \& Bourgin, P. Efficacy of light therapy versus antidepressant drugs, and of the combination versus monotherapy, in major depressive episodes: A systematic review and meta-analysis. Sleep Med Rev, 48, 101213 (2019).

7. Al-Karawi, D. \& Jubair, L. Bright light therapy for nonseasonal depression: Meta-analysis of clinical trials. J Affect Disord, 198, 64-71 (2016).

8. Bassa, D., Canazei, M., Hinterhuber, H. \& Weiss, E. M. Lichttherapie: zum Stand der aktuellen Forschung. Neuropsychiatr, 27, 142-148 (2013).

9. Kogan, A. O. \& Guilford, P. M. Side effects of short-term 10,000-lux light therapy. Am J Psychiatry, 155(2), 293-294 (1998).

10. Terman, M., Schlager, D., Fairhurst, S. \& Perlman, B. Dawn and dusk simulation as a therapeutic intervention. Biol Psychiatry, 25, 966-970 (1989).

11. Avery, D. H. et al. Dawn simulation and bright light in the treatment of SAD: a controlled study. Biol Psychiatry, 50 (3), 205-216 (2001).

12. Terman, M. \& Terman, J. S. Controlled trial of naturalistic dawn simulation and negative air ionization for seasonal affective disorder. Am J Psychiatry, 163 (12), 2126-2133 (2006).

13. Danilenko, K. V. \& Ivanova, I. A. Dawn simulation vs. Bright light in seasonal affective disorder: treatment effects and subjective preference. J Affect Disord, 180, 87-89 (2015).

14. Arakawa, K. et al. Effects of the gradually increasing dawn light stimulation on sleep feeling. Psychiatry Clin Neurosci, 52 (2), 247-248 (1998). 
15. Thompson, A., Jones, H., Gregson, W. \& Atkinson, G. Effects of dawn simulation on markers of sleep inertia and post-waking performance in humans. Eur J Appl Physiol, 114, 1049-1056 (2014).

16. Van De Werken, M. et al. Effects of artificial dawn on sleep inertia, skin temperature, and the awakening cortisol response. J Sleep Res, 19 (3), 425-435 (2010).

17. Gabel, V. et al. Dawn simulation light impacts on different cognitive domains under sleep restriction. Behav Brain Res, 281, 258-266 (2015).

18. Danilenko, K. V. et al. Phase advance after one or three simulated dawns in humans. Chronobiol Int, 17 (5), 659-668 (2000).

19. Viola, A. U. et al. Dawn simulation light: a potential cardiac events protector. Sleep Med, 16 (4), 457461 (2015).

20. Daan, S. et al. Assembling a clock for all seasons: are there $M$ and $E$ oscillators in the genes? J Biol Rhythms, 16 (2), 105-116 (2001).

21. Danilenko, K. V. \& Hommes, V. Influence of artificial dusk on sleep. Sleep Biol. Rhythms, 14, 47-53 (2016).

22. Fontana-Gasio, P. et al. Dawn-dusk simulation light therapy of disturbed circadian rest-activity cycles in demented elderly. Exp Gerontol, 38 (1-2), 207-216 (2003).

23. Bromundt, V. et al. Effects of a dawn-dusk simulation on circadian rest-activity cycles, sleep, mood and well-being in dementia patients. Exp Gerontol, 124, 110641 (2019).

24. Rahman, S. A., St Hilaire, M. A. \& Lockley, S. W. The effects of spectral tuning of evening ambient light on melatonin suppression, alertness and sleep. Physiol Behav, 177, 221-229 (2017).

25. Santhi, N. et al. The spectral composition of evening light and individual differences in the suppression of melatonin and delay of sleep in humans. J Pineal Res, 53 (1), 47-59 (2012).

26. Vethe, D. et al. The evening light environment in hospitals can be designed to produce less disruptive effects on the circadian system and improve sleep. Sleep, 44 (3), zsaa194 (2021).

27. Chellappa, S. L. et al. Acute exposure to evening blue-enriched light impacts on human sleep. J Sleep Res, 22 (5), 573-580 (2013).

28. Hester, L. et al. Evening wear of blue-blocking glasses for sleep and mood disorders: a systematic review. Chronobiol Int, 24, 1-9 (2021).

29. Spitschan, M. Melanopsin contributions to non-visual and visual function. Current Opinion in Behavioral Sciences, 30, 67-72 (2019).

30. Lyall, L. M. et al. Association of disrupted circadian rhythmicity with mood disorders, subjective wellbeing, and cognitive function: a cross-sectional study of $91 t^{\wedge} 105$ participants from the UK Biobank. Lancet Psychiatry, 5 (6), 507-514 (2018).

31. Martin, J. L. \& Hakim, A. D. Wrist actigraphy. Chest, 139 (6), 1514-1527 (2011).

32. Gonçalves, B. S., Adamowicz, T., Louzada, F. M., Moreno, C. R. \& Araujo, J. F. A fresh look at the use of nonparametric analysis in actimetry. Sleep Med Rev, 20, 84-91 (2015). 
33. Marino, M. et al. Measuring sleep: accuracy, sensitivity, and specificity of wrist actigraphy compared to polysomnography. Sleep, 36 (11), 1747-1755 (2013).

34. Ancoli-Israel, S. et al. The role of actigraphy in the study of sleep and circadian rhythms. Sleep, 26 (3), 342-392 (2003).

35. Blume, C., Santhi, N. \& Schabus, M. 'nparACT' package for R: A free software tool for the nonparametric analysis of actigraphy data. MethodsX, 24 (3), 430-435 (2016).

36. Hayasaka, Y. et al. Dose equivalents of antidepressants: Evidence-based recommendations from randomized controlled trials. J Affect Disord, 180, 179-184 (2015).

37. Leucht, S., Samara, M., Heres, S. \& Davis, J. M. Dose Equivalents for Antipsychotic Drugs: The DDD Method. Schizophr Bull, 42(Suppl (1), 90-94 (2016).

38. Inada, T. \& Inagaki, A. Psychotropic dose equivalence in Japan. Psychiatry Clin Neurosci, 69 (8), 440-447 (2015).

39. Knapen, S. E., van derWerken, M., Gordijn, M. C. \& Meesters, Y. The duration of light treatment and therapy outcome in seasonal affective disorder. J Affect Disord, 166, 343-346 (2014).

40. World Health Organization. ICD-10: international statistical classification of diseases and related health problems: tenth revision 2nd edn (World Health Organization, 2004).

41. Wittchen, H. U., Zaudig, M. \& Fydrich, T. Strukturiertes Klinisches Interview für DSM-IV (Hogrefe, 1997).

42. Hautzinger, M., Bailer, M., Worall, H. \& Keller, F. Beck-Depressions-Inventar (BDI)(Huber, 1994).

43. Faul, F., Erdfelder, E., Lang, A. G. \& Buchner, A. G*Power 3: A flexible statistical power analysis program for the social, behavioral, and biomedical sciences. Behavior Research Methods, 39, 175191 (2007).

44. Cohen, J. Statistical Power Analysis for the Behavioral Sciences 2nd edn (Lawrence Erlbaum Associates, 1988).

45. Fang, H., Tu, S., Sheng, J. \& Shao, A. Depression in sleep disturbance: A review on a bidirectional relationship, mechanisms and treatment. J Cell Mol Med, 23 (4), 2324-2332 (2019).

46. Difrancesco, S., Penninx, B. W., Riese, H., Giltay, E. J. \& Lamers, F. The role of depressive symptoms and symptom dimensions in actigraphy-assessed sleep, circadian rhythm, and physical activity. Psychol Med, 12, 1-7 (2021).

47. Tazawa, Y. et al. Actigraphy for evaluation of mood disorders: A systematic review and metaanalysis. J Affect Disord, 253, 257-269 (2019).

48. Bunney, W. E. \& Bunney, B. G. Molecular clock genes in man and lower animals: possible implications for circadian abnormalities in depression. Neuropsychopharmacology, 22 (4), 335-345 (2000).

49. Minaeva, O. et al. Level and timing of physical activity during normal daily life in depressed and nondepressed individuals. Transl Psychiatry, 10 (1), 259 (2020).

50. Terman, M. Evolving applications of light therapy. Sleep Med Rev, 11 (6), 497-507 (2007).

51. George, S. V., Kunkels, Y. K., Booij, S. \& Wichers, M. Uncovering complexity details in actigraphy patterns to differentiate the depressed from the non-depressed. Sci Rep. 11(1), 13447(2021). 
52. Burton, C. et al. Activity monitoring in patients with depression: a systematic review. J Affect Disord, 145 (1), 21-28 (2013).

53. Maruani, J. \& Geoffroy, P. A. Bright Light as a Personalized Precision Treatment of Mood Disorders. Front Psychiatry, 10, 85 (2019).

54. Malhi, G. S., Bell, E., Morris, G. \& Hamilton, A. The delay in response to antidepressant therapy: A window of opportunity? Aust N Z J Psychiatry, 54 (2), 127-129 (2020).

55. Agid, O., Kapur, S., Arenovich, T. \& Zipursky, R. B. Delayed-onset hypothesis of antipsychotic action: a hypothesis tested and rejected. Arch Gen Psychiatry, 60 (12), 1228-1235 (2003).

56. Valenstein, M. et al. Benzodiazepine use among depressed patients treated in mental health settings. Am J Psychiatry, 161 (4), 654-661 (2004).

57. Ogawa, Y. et al. Antidepressants plus benzodiazepines for adults with major depression. Cochrane Database Syst Rev, 6 (6), CD001026 (2019).

58. Dell'osso, B. \& Lader, M. Do benzodiazepines still deserve a major role in the treatment of psychiatric disorders? A critical reappraisal. Eur Psychiatry, 28 (1)), 7-20 (2013).

59. UK ECT Review Group. Efficacy and safety of electroconvulsive therapy in depressive disorders: a systematic review and meta-analysis. Lancet, 361 (9360), 799-808 (2003).

60. loannou, M. et al. Sleep deprivation as treatment for depression: Systematic review and metaanalysis. Acta Psychiatr Scand, 143 (1), 22-35 (2021).

61. Tao, L. et al. Light therapy in non-seasonal depression: An update meta-analysis. Psychiatry Res, 291, 113247 (2020).

62. Beauchemin, K. M. \& Hays, P. Sunny hospital rooms expedite recovery from severe and refractory depressions. J Affect Disord, 40 (1-2), 49-51 (1996).

63. Canellas, F. et al. Increased daylight availability reduces length of hospitalisation in depressive patients. Eur Arch Psychiatry Clin Neurosci, 266 (3), 277-280 (2016).

64. Gbyl, K. et al. Depressed Patients Hospitalized in Southeast-Facing Rooms Are Discharged Earlier than Patients in Northwest-Facing Rooms. Neuropsychobiology, 74 (4), 193-201 (2016).

65. Benedetti, F., Colombo, C., Barbini, B., Campori, E. \& Smeraldi, E. Morning sunlight reduces length of hospitalization in bipolar depression. J Affect Disord, 62 (3), 221-223 (2001).

\section{Figures}




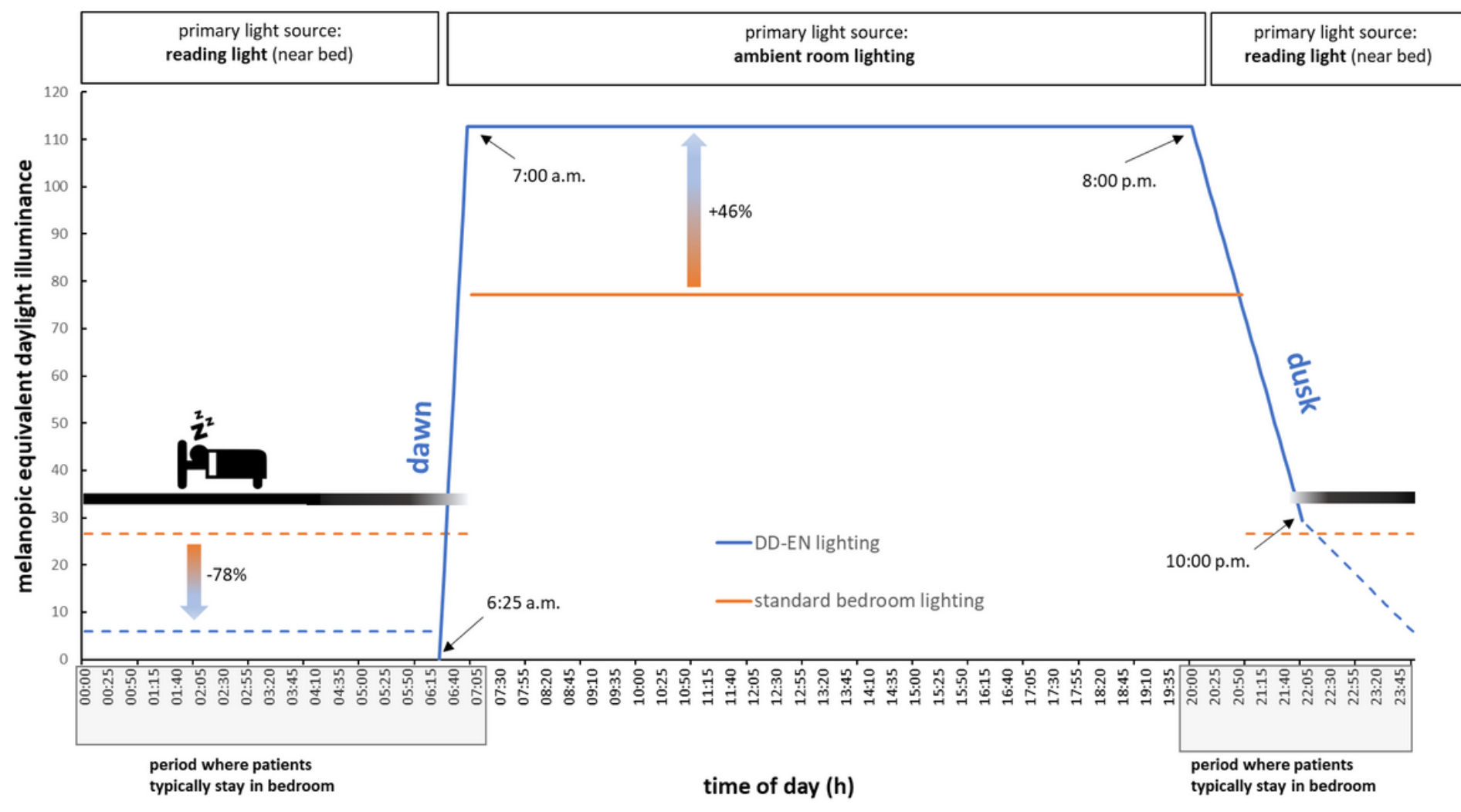

Figure 1

Light control strategy for the DD-N bedroom lighting system. 


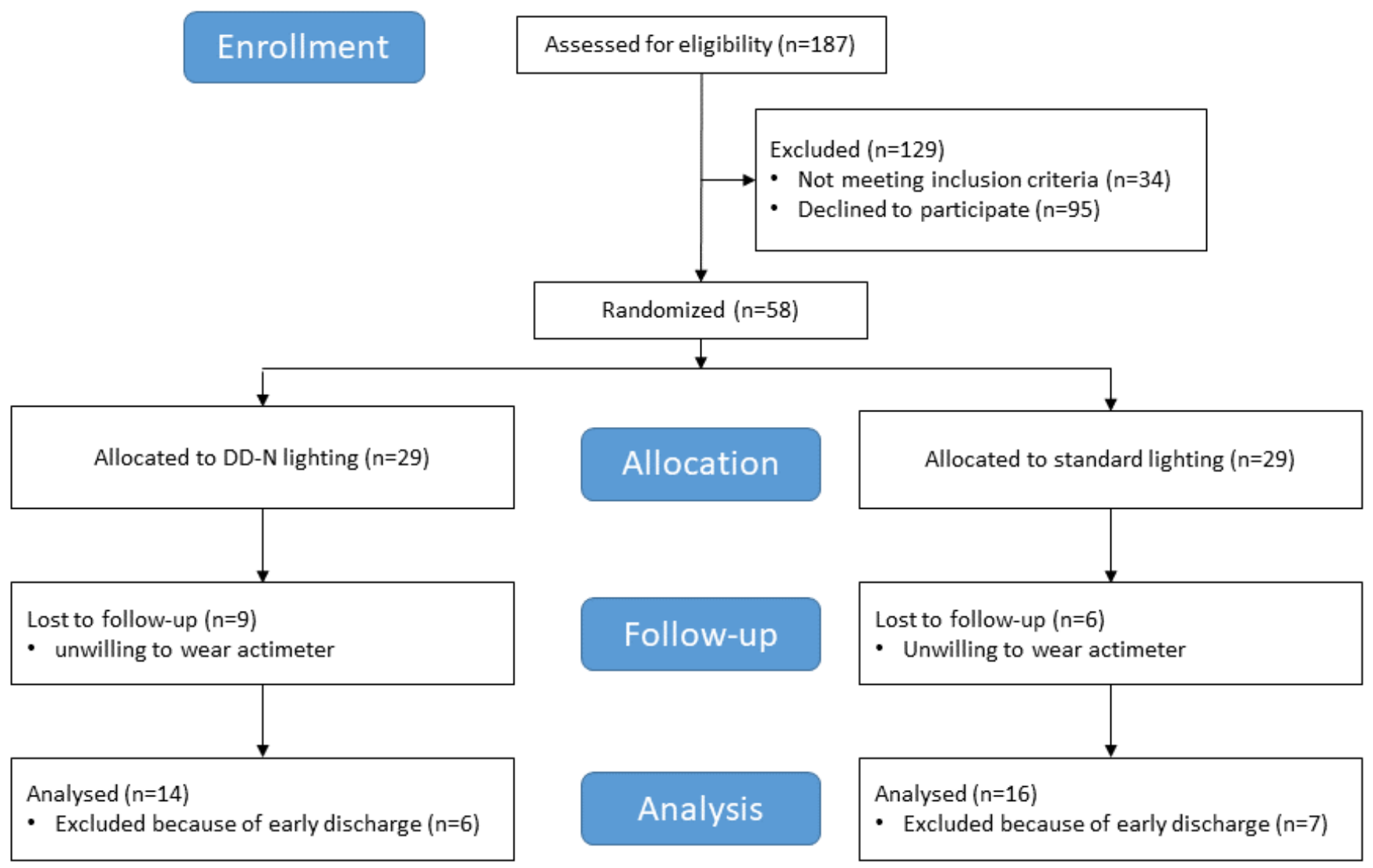

Figure 2

Study flow diagram. 


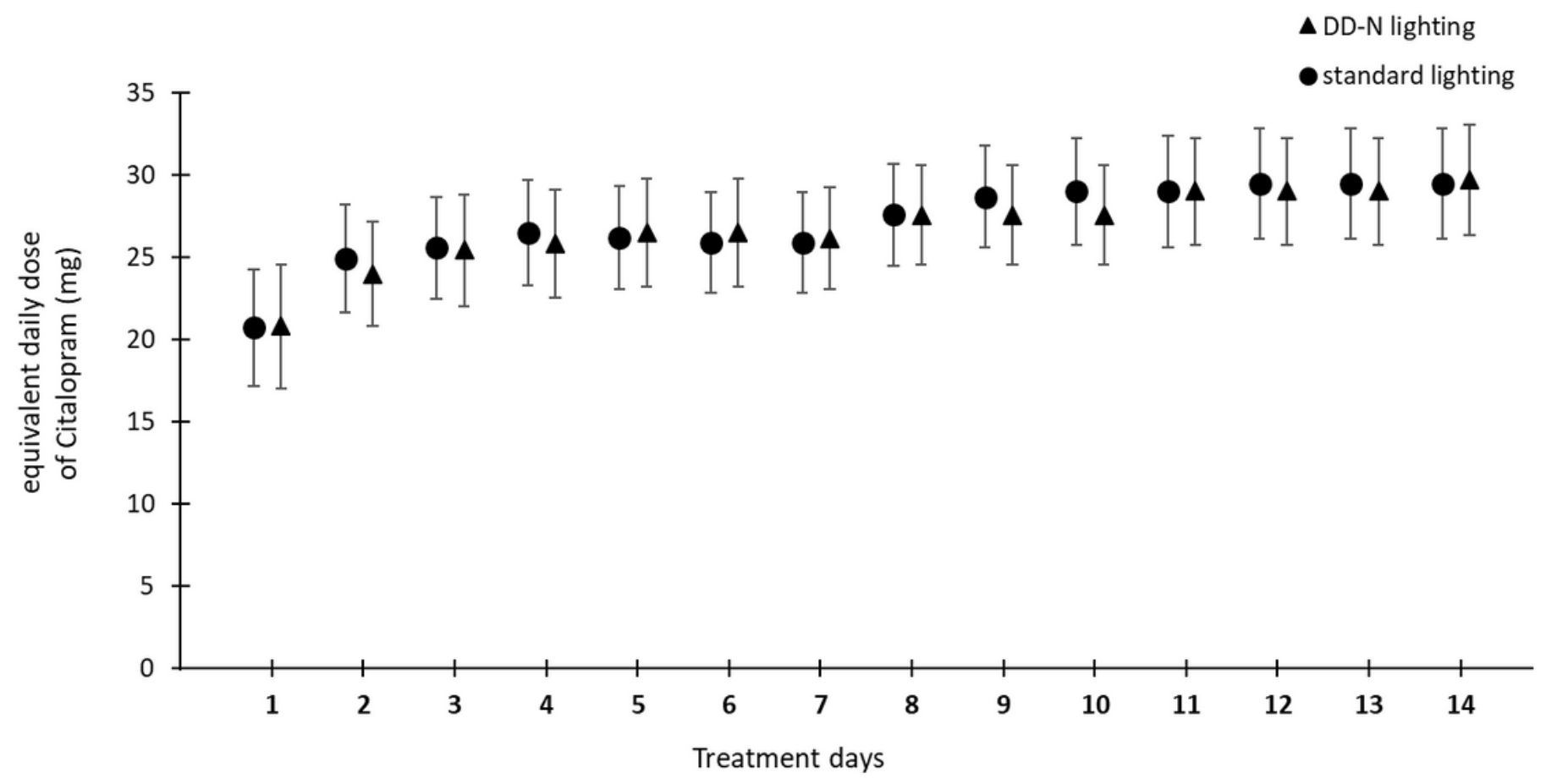

Figure 3

Daily antidepressant medication.

\section{Supplementary Files}

This is a list of supplementary files associated with this preprint. Click to download.

- supplementarymaterials.docx 